\title{
The Orbital Design and Control Strategy of Chang'e-3 Soft-landing
}

\section{Huang Xinyi}

\author{
North China Electric Power University, Hebei, Baoding071003
}

\begin{abstract}
We take the orbital design and control strategy of Chang'e-3 soft-landing as the research object in this paper. First we should establish the three-dimension dynamical model of uniform sphere and the dynamical model of soft-landing under the selenocentric inertial system, then we adopt a new optimization algorithm, namely Ant Colony Algorithm, ACA, to study the lunar soft-landing trajectory optimization problem, and calculate the Chang'e-3 landing orbit. Finally, we use Hohmann transfer to figure out the total time of six stages first, and we use the MATLAB software simulation and calculate the Moon landing site step by step. Then we use the Excel software to fit the 6 stages so as to calculate the corresponding time of the 6 stages respectively. Based on the detailed analysis of Chang'e-3 landing orbit, we adopt covariance analysis to analyze the orbital error caused by the error source of the orbital initial error. We make the complexity of the solving process which is difficult to achieve be reduced, and we evaluate and promote the model, namely, sensitivity analysis, to make it be able to be better used in the actual production and life.
\end{abstract}

Key Words: The Three-dimension Dynamical Model of Uniform Sphere; Ant Colony Algorithm; Hohmann Transfer; Covariance Analysis; MATLAB; Excel Software

\section{Introduction}

This paper gives that the operation quality of Chang'e-3 is $2.4 \mathrm{t}$ when it is on the orbit prepared for the landing, the main speed-down motor installed on the bottom of it is able to produce the adjustable thrust with $1500 \mathrm{~N}$-$7500 \mathrm{~N}$, and its specific impulse (namely, the thrust produced by the propellant per unit mass) is $2940 \mathrm{~m} / \mathrm{s}$ which can meet the control requirements of the adjusting speed. The attitude regulating motor is installed around it. After we give the thrust direction of the main speed-down motor, we can realize the adjustment control of various attitudes automatically by the pulse combination of multiple motors. The scheduled landing site of Chang'e-3 is $19.51 \mathrm{~W}, 44.12 \mathrm{~N}$, and there is - $2641 \mathrm{~m}$ above sea level (Shown in Annex 1) .

The basic requirements for the orbital design of Chang'e-3 landing are as follows. The orbit prepared for the landing is elliptical orbit with the perilune point of $15 \mathrm{~km}$ and the aposelenium point of $100 \mathrm{~km}$. The landing orbit is from the perilune point to the landing site. And it is required to meet the states of each stage (There are six stages in total) at the key points in the process of soft landing and minimize the fuel consumption in the process of soft landing.

According to the basic requirements above, we establish the mathematical model to solve the following problems.

(1) Confirm the position of the perilune point and the aposelenium point with the orbit prepared for the landing, and corresponding 
magnitude and direction of velocity with Chang'e-3.

(2) Confirm the landing orbit and the optimal control strategy in six stages of Chang'e-3.

(3) Do the corresponding error analysis and sensitivity analysis for the designed of landing orbit and control strategy.

\section{Model Assumption}

1. Suppose that we do not consider the influence of the Moon's rotation on latitude and longitude.

2. Suppose that we ignore the Moon's rotation, the nonspherical item of lunar gravity and the influence of lunisolar gravitational perturbation, etc.

3. Suppose that we only consider the nonspherical gravitational perturbation of the Moon, and the gravitational perturbation of the Earth and the Sun.

4. Suppose that we consider the Moon as a sphere to establish uniform sphere.

5. Suppose that other perturbation terms can be ignored on condition that there is no atmosphere when it is landing on the lunar surface and the time of soft landing process is relatively short.

6. Suppose that the moon is a uniform gravitational field in the process of soft landing.

\section{Symbol Description}

A

indicates the transverse selenocentric angle in the XrYr plane; $\beta$ indicates the longitudinal selenocentric angle in declining orbital plane; $r$ indicates the radius vector of the lander; $\alpha_{L}$ indicates the right ascension of the lander; $\beta_{L}$ indicates the declination of the lander; $\mathrm{U}$ indicates the component of declining velocity in Ox axis of the coordinate system; $\mathrm{V}$ indicates the component of declining velocity in Oy axis of the coordinate system; W indicates the component of declining velocity in $\mathrm{Oz}$ axis of the coordinate system; $\rho$ indicates volatilization coefficient; $\mathrm{K} 、 \mathrm{Q}$ indicate adjustment coefficients; $v$ indicates the speed of Chang'e-3; $\mu$ indicates the standard gravity parameter of the central object; a indicates the semi-major axis of its orbit; $\mathrm{V}_{1}$ indicates the running speed of the aphelion; $\mathrm{V}_{2}$ indicates the running speed of the perihelion; $\mathrm{C}_{\mathrm{X}}$ indicates the covariance matrix of the independent variables; $\mathrm{Cy}$ indicates the covariance matrix of the function; * indicates that other contents which are not mentioned will explain in the paper.

\section{The Establishment of the Model and}

\section{Its Solution}

4.1 The Establishment of the Dynamic Model, the Confirmation of the Position of the Perilune Point and the Aposelenium Point with the Orbit Prepared for the Landing and the Confirmation of Corresponding Magnitude and Direction of Velocity with Chang'e-3

This paper first studies the dynamic model of the orbital landing with Chang'e-3 in different stages. Because this is the general analysis of the orbit. We only consider the nonspherical lunar gravitational perturbation and the lunisolar gravitational perturbation. Then, generally the vector expression of lunar softlanding dynamic model can be written as follows. 


$$
r=F-\frac{\mu_{m}}{r^{3}}+F_{\varepsilon}-\mu_{e}\left(\frac{1}{r_{e}^{3}} * r_{e}+\frac{1}{\Delta_{e}^{3}} * \Delta_{e}\right)-\mu_{s}\left(\frac{1}{r_{s}^{3}} r_{s}+\frac{1}{\Delta_{s}^{3}} \Delta_{s}\right)
$$

The distance between the lander and the lunar surface is relatively high. And the distance of the lunar surface which the lander has walked along is longer. Therefore, if we consider the Moon as the plane to establish model, it will bring a large deviation. The braking phase is necessary to consider the Moon as the sphere to establish three-dimensional softlanding model with the uniform sphere. The propulsion system of braking phase adopts constant thrust method, and completes the change for the direction of braking force by attitude control.

\subsubsection{The Three-dimensional Dynamic Model of Uniform Sphere and the Softlanding Dynamic Model with the Selenocenter Inertial System}

First we define some coordinate systems. 1) The reference inertial coordinate system OXrYrZr. The origin $\mathrm{O}$ is located in the center of the Moon. The $\mathrm{Zr}$ axis points from the selenocenter to the initial softlanding point. The $\mathrm{Xr}$ axis is located in the lunar orbit plane and points to forward direction. The $\mathrm{Yr}$ axis, $\mathrm{Xr}$ axis and $\mathrm{Zr}$ axis form the right angle coordinate system. The coordinate system is only used for softlanding descent oribit. 2)The descent oribit reference coordinate system $\mathrm{OxO}$ yO $\mathrm{zO}$. The origin $\mathrm{O}$ is located in the mass center of the lander. The zo axis points from the selenocenter to the mass center of the lander with positive direction. The xo axis is located in the local plane and points to forward direction of the lander. The yo axis, xo axis and zo axis form the right angle coordinate system. 3) The lander body coordinate system $\mathrm{xb} y \mathrm{~b} \mathrm{zb}$. The origin $\mathrm{O}$ is located in the mass center of the lander. The $\mathrm{xb}$ axis is located in the braking thrust vector extension line. It is along the thrust direction with the positive direction. The yb axis, $\mathrm{zb}$ axis are respectively confirmed according to the equipment installation on the lander. And they form the right angle coordinate system together with $\mathrm{xb}$ axis.

In order to be consistent with the reference system of circumlunar movement and at the same time facilitate the analysis of softlanding descent window, we need to make the motion of the lander be indicated in the selenocentric equatorial inertial coordinate system. First we give the defination of the selenocentric equatorial inertial system OXYZ.The origin $\mathrm{O}$ is located in the center of the Moon. The XY plane is located in the lunar equatorial plane. Among them, the $\mathrm{X}$ axis points to the projection of J2000 mean equinox on the lunar equator. Z axis points to the Moon's North Pole. Y axis, X axis and $\mathrm{Z}$ axis form the right angle coordinate system.

And ultimately we caculate the position of perihelion with the longitude $19.256 \mathrm{~W}$, the latitude $37.434 \mathrm{~N}$, and $15.667 \mathrm{Km}$ above sea level and the position of aphelion with the longitude $160.9536 \mathrm{E}$, the latitude $28.9989 \mathrm{~S}$, and 101.211 Km above sea level. Then we can caculate that the running speed of perihelion is $1.692 \mathrm{Km} / \mathrm{s}$ and the running speed of aphelion is $1.614 \mathrm{Km} / \mathrm{s}$ according to the law of universal gravitation and Kepler's third law. 
4.2 The Confirmation of the Landing Orbit and the Optimal Control Strategy in Six Stages of Chang'e-3

\subsubsection{The Solution of the Landing Orbit with} Ant Colony Algorithm

Because the surface of the moon has no atmosphere, only the speed of the lander must be completely offset by the braking motor we can achieve a soft landing safely. This process needs to consume a large amount of fuel. So it is necessary to do the optimal design for this process. In order to confirm the landing orbit of Chang'e-3, it can be divided into two kinds, namely direct method and indirect method. This paper adopts a new optimization algorithm, namely Ant Colony Algorithm, ACA, to study the orbital optimization problem of lunar soft landing. We get the decimal Ant Colony Algorithm which is suitable for continuous parameter optimization problem by improving the basic Ant Colony Algorithm.

4.2.2 Comparison and Description of the Optimal Solution Got by Using Ant Colony Algorithm

Table 1 shows the comparative result between the Ant Colony Algorithm and the simulated annealing genetic algorithm. The simulated annealing genetic algorithm is a kind of hybrid algorithm got by combining adaptive genetic algorithm and simulated annealing algorithm. The data in the table are the average values of the results got by calculating ten times with the two kinds of algorithms.

Table 1 The Comparative Chart between the Ant Colony Algorithm and the Simulated Annealing Genetic Algorithm

\begin{tabular}{|c|c|c|c|}
\hline & $\begin{array}{c}\text { The Result of the Ant } \\
\text { Colony Algorithm }\end{array}$ & $\begin{array}{c}\text { The Result of the } \\
\text { Simulated Annealing } \\
\text { Genetic Algorithm }\end{array}$ & Constraint Values \\
\hline $\begin{array}{c}\text { The Average Time } \\
\text { Length of Spray Jet/s }\end{array}$ & 608.4 & 607.9 & absence of restriction \\
\hline $\begin{array}{c}\text { The Average Fuel } \\
\text { Consumption /Kg }\end{array}$ & 278.78 & 279.01 & absence of restriction \\
\hline $\begin{array}{c}\text { Radial Velocity of the } \\
\text { Average Landing } \\
/(\mathrm{m} / \mathrm{s})\end{array}$ & -0.0013587 & -0.0014592 & 1738000 \\
\hline $\begin{array}{c}\text { Selenocentric Distance } \\
\text { of the Average } \\
\text { Landing/m }\end{array}$ & 1738000.045 & 1737998.978 & 0 \\
\hline $\begin{array}{c}\text { Polar Angle Angular } \\
\text { Velocity of the } \\
\text { Average Landing/ } \\
\text { (rad/s })\end{array}$ & $4.214 \mathrm{e}-024$ & $4.478 \mathrm{e}-030$ & \\
\hline $\begin{array}{c}\text { The Average } \\
\text { Optimization Time/min }\end{array}$ & 42 & 245 & absence of restriction \\
\hline
\end{tabular}




\subsubsection{The Calculated Time of Horman Transfer}

The optimal problem of fuel consumption in fact is time optimal problem. Therefore, this paper firstly uses Hohmann transfer to caculate the landing time of Chang'e-3, then confirms the optimal control strategy with 6 stages.

For the coarse obstacle avoidance section, its scope is the interval between $2.4 \mathrm{~km}$ and $100 \mathrm{~m}$ from the lunar surface. And the main requirement is to avoid the large craters to achieve the hovering with $100 \mathrm{~m}$ above the designed landing point, and confirm the moon landing site initially. For fine obstacle avoidance section, its interval is $100 \mathrm{~m}-30 \mathrm{~m}$ from the lunar surface. We require Chang'e-3 to hover at the site with $100 \mathrm{~m}$ from lunar surface. We use MATLAB software to confirm the best landing site to achieve the horizontal velocity, $0 \mathrm{~m} / \mathrm{s}$, with $30 \mathrm{~m}$ above the landing point.

The initial conditions in this paper is given by the perilune point with $100 \mathrm{x} 15 \mathrm{~km}$ Hohmann transfer orbit. Suppose that the Moon is a regular sphere, and its radius is $\mathrm{Rm}=$ $1738 \mathrm{~km}$, its gravitational constant is $\mu=$ $4902175 \mathrm{~km}^{3} / \mathrm{s}^{2}$. The softlanding boundary value conditions are given as follows: $r_{0}=$ $1753 \mathrm{~km}, \mathrm{r}_{\mathrm{f}}=1740 \mathrm{~km} ; \mathrm{v}_{0}=1.6922 \mathrm{~km} / \mathrm{s}, \mathrm{v}_{\mathrm{f}}=0$. The brake motor thrust $\mathrm{F}=1500 \mathrm{~N}$, and specific impulse Isp $=2940 \mathrm{~m} / \mathrm{s}$. According to the above formula, we use Excel software to fit the six stages so as to work out the corresponding time for each section. Therefore, the corresponding time for each stage is shown respectively as follows. Main reduction section is 487s. Quick adjustment section is 34s. Coarse obstacle avoidance section is 146s. Fine obstacle avoidance section is $29 \mathrm{~s}$. And Slow decline section is $24 \mathrm{~s}$.

\subsection{Doing the Corresponding Error Analysis and Sensitivity Analysis of Landing Orbit and Control Strategy}

Based on the detailed analysis of the chang 'e 3 landing orbit, this paper adopts covariance analysis method to analyze the orbital error caused by the error source of the orbital initial error. It analyzes that the initial orbit position error and initial velocity error have an impact on the position of the orbital end point and the velocity error.

The basic principle of covariance analysis method

For the nonlinear function relationship y $=\mathrm{f}(\mathrm{x} 1, \mathrm{x} 2 \ldots \mathrm{Xn})$, we can use the first-order Taylor series to linearize it. So there are the following formula.

$$
y+\Delta y=f+\frac{\partial f}{\partial x_{1}} \Delta x_{1}+\frac{\partial f}{\partial x_{n}} \Delta x_{n}+o\left(x_{1} \cdots x_{n}\right)
$$

In them, $o\left(x_{1} \cdots x_{n}\right)$ is the higher order term of $x 1, x 2 \cdots x n$.

So, we can get the linearized equation, $\delta y=\sum_{i=1}^{n} \frac{\partial f}{\partial x_{i}} \delta x_{i}$

If the independent variable $\delta x_{1} \cdots \delta x_{n}$ is a random variable, the covariance matrix with the function $\delta y$ of linear equation is $E\left(\delta Y^{*} \delta Y^{T}\right)=E\left(P \delta X \delta X^{T} P^{T}\right)=P E\left(\delta X \delta X^{T}\right) P^{T}$

, namely, $C x=P C x P^{T}$. In this equation, $\mathrm{C}_{\mathrm{X}}$ is the covariance matrix of the independent variable, and $\mathrm{Cy}$ is the covariance matrix of the function $\delta y$. 
The diagonal element is variance and the non-diagonal element is covariance in the covariance matrix. Obviously, as long as we caculate the transfer matrix $P$ we can confirm the relationship between source error and desire amount error. If we are given various source errors, such as the motor installation error, the sensors measurement error or the motor thrust error and ignition time error and other errors, we can analyze the influence on the target orbit error and the influence on the precision of the control system to further put forward appropriate requirements with precision of each system and component.

The error sources which cause the orbit errors are mainly the main reducer thrust error, including position error and velocity error. In them, the position error is $\Delta r=\left(\Delta r_{x}, \Delta r_{y}, \Delta r_{z}\right)$, and $\Delta r_{x}, \Delta r_{y}, \Delta r_{z}$ are respectively the components of $\mathrm{X}$ axis, $\mathrm{Y}$ axis and $\mathrm{Z}$ axis in geocentric inertial coordinate system. The velocity error is $\Delta v=\left(\Delta v_{x}, \Delta v_{y}, \Delta v_{z}\right)$, and
$\Delta v_{x}, \Delta v_{y}, \Delta v_{z}$ are the components of $\mathrm{X}$ axis, $\mathrm{Y}$ axis and $\mathrm{Z}$ axis in geocentric inertial coordinate system.

The initial orbit position and velocity error of the chang 'e 3 landing orbit are determined by the precision for the carrier rocket that carries it entering the orbit. If the detector does orbital correction in flight, the orbital position error after the orbital correction will be determined by the navigation error and the velocity error will bedetermined by the attitude error and the guidance error.

The errors above determine the initial conditions with calculation of the orbital error covariance analysis. We use Excel software to fit corresponding orbital positions with the landing process of the detector and the time course of the total velocity error. I

Finally we calculate that the initial orbit position error and initial velocity error have an impact on the position of the orbital end point and the velocity error in geocentric inertial coordinate system when we don't do orbital correction in flight. As shown in table 2.

Table 2 The Influence Chart for the Influence on the Position of the Orbital End Point by the Initial Orbit Position Error and Initial Velocity Error

\begin{tabular}{|c|c|c|c|c|c|c|c|c|c|c|c|}
\hline \multicolumn{4}{|c|}{ The Orbit Initial Error } & \multicolumn{5}{c|}{ The Orbit End Point Error } \\
\hline \multicolumn{2}{|c|}{ Position Error/Km } & \multicolumn{2}{|c|}{ Velocity Error m/s } & \multicolumn{3}{|c|}{ Position Error/Km } & \multicolumn{2}{|c|}{ Velocity Error m/s } \\
\hline$\Delta r_{x}$ & $\Delta r_{y}$ & $\Delta r_{z}$ & $\Delta v_{x}$ & $\Delta v_{y}$ & $\Delta v_{z}$ & $\Delta r_{x}$ & $\Delta r_{y}$ & $\Delta r_{z}$ & $\Delta v_{x}$ & $\Delta v_{y}$ & $\Delta v_{z}$ \\
\hline 1.0 & 1.0 & 1.0 & 0.0 & 0.0 & 0.0 & 723.4 & 632.2 & 75.3 & 3.95 & 3.64 & 0.52 \\
\hline
\end{tabular}




\section{The Evaluation and Improvement of}

\section{the Model}

\subsection{Model Evaluation}

First, this paper establishes the three-dimensional dynamic model of the uniform sphere and softlanding dynamic model of the selenocentric inertial system, and calculate perihelion position and aphelion position of the orbit prepared for the landing. And then we use Kepler's third law to calculate the speed of perihelion and aphelion in this paper. This model method is relatively simple and it has high accuracy. And the maneuverability is strong.

Second, this paper adopts a kind of new optimization algorithm, namely Ant Colony Algorithm, ACA, to study the the lunar softlanding orbital optimization problem so as to caculate the chang 'e 3 landing orbit. Then we use the comparison method to describe this algorithm to calculate the optimal landing orbit.

Next, we use Hohmann transfer to caculate the total time of six stages and use the MATLAB software to simulate it to caculate the Moon landing position. At last we use Excel software to fit the six stages so that we can caculate the respective corresponding time for the six stages.

Finally, based on the detailed analysis of

$$
\begin{aligned}
& \operatorname{Max} \quad \mathrm{X}=\sum_{J=1}^{N} C_{J} X_{J}
\end{aligned}
$$

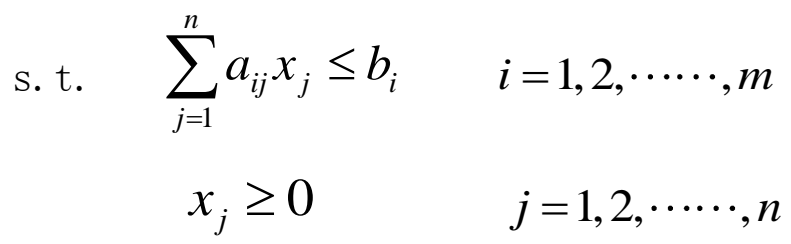

The error is usually \pm 0.1 . the chang 'e 3 landing orbit, this paper adopts the covariance analysis method to analyze the orbital error caused by the error sources of the orbital initial error. And we use Excel software to make fitting analysis. We calculate finally that the initial orbit position error and initial velocity error have an impact on the position of the orbital end point and the velocity error.

However,there may be small errors when the program is running and fitting. What's more, there may be minor differences with the true model when the model is selected.And it will also have a certain impact on the caculated result.

\subsection{Sensitivity Analysis}

In the analysis of project, people not only care about the performance prediction of the products but also concerne about changing the design with the result of the performance prediction and using the sensitivity for correcting errors to analyze. We analyze the sensitivity of these parameters to make quantitative analysis which is called sensitivity analysis. In this paper, we make other conditions be unchanged and make the quality of the chang 'e 3 be changed from $2.4 \mathrm{t}$ to $3 \mathrm{t}$ to test the landing orbit whether gets bigger or not. For linear problems, 


\subsection{Improvement and Promotion}

This dynamic model which is established in this paper has a wide applied range and it can be applied to the problem of natural resources consumption, the problems of the intensity and magnitude with earthquake and other aspects. And it is worth promoting.

\section{6.conclusions}

In terms of problems, we calculate that the initial orbit position error and initial velocity error have an impact on the position of the orbital end point and the velocity error in geocentric inertial coordinate system when we don't do orbital correction in flight, In the analysis of project, people not only care about the performance prediction of the products but also concerned about changing the design with the result of the performance prediction and using the sensitivity for correcting errors to analyze.

\section{Reference}

[1]Fu Jialiang. Operations Research Method and Model. Shanghai: Fudan University Publishing House, 2006.288-289
[2]Wu Qizong. Systems Engineering. Beijing: Beijing Institute Technology Press, 2008.69-72. [3]Cao Yan. MATLAB R2008 Mathematics and Control Instance Tutorial. Beijing:Chemical Industry Publishing House, 2009. [4]Zheng A'qi. MATLAB Practical Tutorial[M]. 2nd Edition. Beijing: Publishing House of Electronics Industry,2005.

[5]Shao Yi. The Calculation Method with Consumers of Optimal Selection Problem under the Condition of Budget Constraint[J].Journal of Hebei Vocational and Technical Normal College, 2002.12 (16): 53-55.

[6]Dong Zhenpu. Mathematical Modeling Methods and Practices[M]. 1st Edition. Beijing:China National Defense Industry Press,2006.

[7]Wikipedia. Orbital Element. http://en.wikipedia.Org/wiki/Orbital_elements. 2014.1.17

[8] Wikipedia. Specific Impulse. http://en.wikipedia.Org/wiki/Specific_impulse.2 014.1.17 